\title{
A Commutation Theorem and Duality for Free Bose Fields
}

\author{
Marc A. Rieffel \\ Department of Mathematics, University of California, Berkeley, California, USA
}

Received May 9; in revised form May 23, 1974

\begin{abstract}
We first present an elementary commutation theorem for operator algebras on Hilbert space. This theorem is then applied to give an elementary proof of duality for free Bose fields.
\end{abstract}

\section{Introduction}

In the approach to quantum field theory which employs von Neumann algebras of local observables (see [10] and references therein), the property of duality states that the commutant of the algebra associated to a nice region in space-time is exactly the algebra associated to the space-like complement of the region. This property has played an important role in recent work of Doplicher, Haag and Roberts concerning the relation between fields, observables, gauge groups and superselection sectors (see $[6,7]$ and the references therein). The first proof that duality holds for free Bose fields was given by Araki $[1,2]$. Another proof, using infinite tensor products, was given by Dell Antonio [4]. A somewhat simplified version of Araki's proof was then given by Osterwalder [15], and, most recently, Eckmann and Osterwalder have given another proof by applying Tomita-Takesaki theory [9]. In this paper we give a yet simpler proof of duality. Our proof consists of first proving a fairly general commutation theorem for operator algebras, which was motivated in part by Dixmier's commutation theorem for quasi-Hilbert algebras [5]. We then show that our commutation theorem can be applied to the Fock representation to prove duality. Our commutation theorem may conceivably be useful in proving duality in more complicated situations.

\section{The Commutation Theorem}

If $A$ is a *-algebra of bounded operators on a Hilbert space, then we will let $A^{\prime}$ denote the (von Neumann) algebra of all those bounded operators which commute with every element of $A$. We write $A^{\prime \prime}$ for $\left(A^{\prime}\right)^{\prime}$. 
The Commutation Theorem. Let $V$ be a Hilbert space, and let $A$ and $B$ be *algebras of bounded operators on $V$. Assume that $A$ and $B$ commute, that is, $a b=b a$ for all $a \in A, b \in B$, and assume that the *algebra, $C$, generated by $A$ and $B$ has a cyclic vector, $w$. Then $A^{\prime}=B^{\prime \prime}$ (and so $B^{\prime}=A^{\prime \prime}$ ) if the following condition is satisfied:

Coupling Condition: If $x$ is in the closure of $A^{\prime} w$ (or we could use $B^{\prime} w$ instead) and if

1) $\langle x, a w\rangle=+\left\langle a^{*} w, x\right\rangle$ for all $a \in A$

2) $\langle x, b w\rangle=-\left\langle b^{*} w, x\right\rangle$ for all $b \in B$, then $x=0$.

In this case the strong operator closure of $A$ will contain the identity operator on $V$, and similarly for $B$.

Conversely, if $A^{\prime}=B^{\prime \prime}$, and if the strong operator closures of $A$ and $B$ contain the identity operator on $V$, then the Coupling Condition is satisfied for any $x$ in the closure of $A^{\prime} w+B^{\prime} w$.

It should quickly be remarked that in most potential applications one will probably not know much about $A^{\prime}$ and $B^{\prime}$, and so the situation in which this theorem will probably be most useful is that in which $A w+B w$ is already dense in $V$, so that one needs to check the Coupling Condition for all $x \in V$. At any rate this will be the case in the application discussed in the next section.

We obtained the proof of the fact that the Coupling Condition implies commutation by trying to imitate the proof of Dixmier's commutation theorem for quasi-Hilbert algebras (p. 70 of [5]). In particular, the Coupling Condition plays a role analogous to that of axiom v) in Dixmier's definition of a quasi-Hilbert algebra. Although it is not entirely explicit in Dixmier's proof, this role consists of ensuring that operators in $A^{\prime}$ can be approximated by operators in $B$ in the strong-* operator topology (p. 20 of [19]) obtained from a sufficiently large set of vectors (namely a cyclic vector in our case). Our theorem is also fairly closely related to Lemma 5.2 of [20]. It will be shown elsewhere [17] that our theorem can be used to give a quite simple proof of the commutation theorem for tensor products of von Neumann algebras.

Proof. We assume first that the Coupling Condition is satisfied. Let $t \in A^{\prime}$. We show that the Coupling Condition ensures that there is a sequence $\left\{b_{n}\right\}$ in $B$ such that

$$
b_{n} w \rightarrow t w \text { and } \quad b_{n}^{*} w \rightarrow t^{*} w .
$$

(strong-* convergence on $w$ ). To this end, let $\tilde{V}$ denote $V$ with complex conjugate structure (p. 9 of [5]), and for $v \in V$ let $\tilde{v}$ denote the corresponding element of $\tilde{V}$. On $V \times \tilde{V}$ define an inner product by

$$
\left\langle\left(u_{1}, \tilde{v}_{1}\right),\left(u_{2}, \tilde{v}_{2}\right)\right\rangle=\left\langle u_{1}, u_{2}\right\rangle+\left\langle v_{2}, v_{1}\right\rangle,
$$


so that $V \times \tilde{V}$ becomes a Hilbert space. Let

and

$$
K=\left\{\left(s w,\left(s^{*} w\right)^{\sim}\right): s \in A^{\prime}\right\}
$$

$$
L=\left\{\left(b w,\left(b^{*} w\right)^{\sim}\right): b \in B\right\} .
$$

Then $K$ and $L$ are linear submanifolds of $V \times \tilde{V}$, and $L$ is contained in $K$. Since $\left(t w,\left(t^{*} w\right)^{\sim}\right)$ is in $K$, it suffices to show that $L$ is dense in $K$.

Let $\bar{K}$ denote the closure of $K$ in $V \times \tilde{V}$. If $L$ is not dense in $K$, then there is a non zero element, $(x, \tilde{y})$, of $\bar{K}$ which is orthogonal to $L$, that is, such that

$$
\langle x, b w\rangle=-\left\langle b^{*} w, y\right\rangle \text { for all } b \in B .
$$

Since $(x, \tilde{y})$ is in $\bar{K}$, there is a sequence, $\left\{s_{n}\right\}$, of elements of $A^{\prime}$ such that

$$
s_{n} w \rightarrow x, \quad s_{n}^{*} w \rightarrow y .
$$

Then for all $a \in A$ we have

$$
\begin{aligned}
& \langle x, a w\rangle=\lim \left\langle s_{n} w, a w\right\rangle \\
& =\lim \left\langle w, s_{n}^{*} a w\right\rangle=\lim \left\langle a^{*} w, s_{n}^{*} w\right\rangle \\
& =\left\langle a^{*} w, y\right\rangle .
\end{aligned}
$$

Replacing $a$ and $b$ by $a^{*}$ and $b^{*}$ and taking complex conjugates of the above equations, we find that both $x+y$ and $x-y$ satisfy Eqs. (1) and (2) of the Coupling Condition. Furthermore $x+y$ and $x-y$ are both in the closure of $A^{\prime} w$. Since we are assuming that the Coupling Condition holds, it follows that $x=0=y$. Thus $L$ is dense in $K$.

Now let $t \in A^{\prime}, r \in B^{\prime}, b, c \in B+\mathbb{C} 1$. Then $c^{*} t b \in A^{\prime}$, so that by the above arguments (with $t$ replaced by $c^{*} t b$ ) there is a sequence $\left\{b_{n}\right\}$ in $B$ such that

$$
b_{n} w \rightarrow c^{*} t b w, \quad b_{n}^{*} w \rightarrow b^{*} t^{*} c w .
$$

Then for $a, d \in A+\mathbb{C} 1$ we have

$$
\begin{aligned}
& \langle r t(a b) w, c d w\rangle=\left\langle r a\left(c^{*} t b w\right), d w\right\rangle \\
& =\lim \left\langle r a\left(b_{n} w\right), d w\right\rangle=\lim \left\langle r a w, d\left(b_{n}^{*} w\right)\right\rangle \\
& =\left\langle r a w, d\left(b^{*} t^{*} c w\right)\right\rangle=\langle\operatorname{tr}(a b w), c d w\rangle .
\end{aligned}
$$

Since $w$ is assumed to be cyclic for $C$, it follows that $t r=r t$, that is, that $A^{\prime} \subseteq B^{\prime \prime}$. Since $A \subseteq B^{\prime}$, we have $A^{\prime}=B^{\prime \prime}$ as desired. (Shoichiro Sakai has pointed out to me that a quite similar argument is used in the proof of Theorem 1 in [18].)

We indicate now why the strong operator closure, $\bar{B}$, of $B$, will contain the identity operator on $V$ (and similarly for $A$ ). Now $\bar{B}$ will contain a maximal projection, namely the supremum of all the projections in $\bar{B}$, 
and this will be the projection onto the closure of $B V$. Thus we must show that $B V$ is dense in $V$. If it is not, let $x$ be the component of $w$ in the orthogonal complement of $B V$. Then, since $B V$ is invariant under $A$, and $w$ is cyclic for $C$, we have $x \neq 0$ and $x \in A^{\prime} w$. Then $x$ satisfies Eqs. (1) and (2), and so the Coupling Condition fails to hold.

Conversely, suppose that the strong operator closures of $A$ and $B$ contain the identity operator on $V$, and that there exists a non-zero $x$ in the closure of $A^{\prime} w+B^{\prime} w$ which satisfies Eqs. (1) and (2) of the Coupling Condition. We will show that $A^{\prime} \neq B^{\prime \prime}$. Now $x$ will not be orthogonal to both $A^{\prime} w$ and $B^{\prime} w$. We give the argument for the case in which $x$ is not orthogonal to $B^{\prime} w$. Otherwise the roles of $A$ and $B$ must be interchanged in the following argument. Now $B^{\prime} w \supseteqq A^{\prime \prime} w$, and if they are not equal then $B^{\prime} \neq A^{\prime \prime}$ and we are done. So we can assume that $x$ is not orthogonal to $A^{\prime \prime} w$. But by von Neumann's double commutant theorem (p. 40 of [5]) $A^{\prime \prime}$ is the strong operator closure of $A$, and so $A w$ is dense in $A^{\prime \prime} w$. Thus we can assume that $x$ is not orthogonal to $A w$.

Let us form the Hilbert space $V \times V$, and let $A$ act on it by $a(u, v)$ $=(a u, a v)$. Then the commutant of this action of $A$ is given by $2 \times 2$ matrices whose entries are easily seen to be in $A^{\prime}$, acting on $V \times V$ in the obvious way. Let $K$ be the subspace in $V \times V$ generated under $A$ by the vector $(w, x)$, and let $P$ be the projection on $K$. Since $K$ is $A$-invariant, $P$ is in the commutant of $A$, and so is given by a matrix

$$
P=\left(\begin{array}{ll}
d & e \\
e^{*} & f
\end{array}\right)
$$

where $d, e, f \in A^{\prime}$, and $0 \leqq d \leqq 1,0 \leqq f \leqq 1$. We will show that $e$ is not in $B^{\prime \prime}$.

Now Eq. (1) of the Coupling Condition can be rewritten as

$$
\langle(x,-w),(a w, a x)\rangle=0
$$

for all $a \in A$. In other words, it says that $(x,-w)$ is orthogonal to $K$, and so $P(x,-w)=0$. In terms of matrix elements we obtain

$$
d x-e w=0 .
$$

Now because the strong operator closure of $A$ contains the identity operator on $V$, we have $(w, x) \in K$, and so $P(w, x)=(w, x)$. Thus we obtain

$$
d w+e x=w .
$$

Suppose that $e$ were in $B^{\prime \prime}$. Now Eq. (2) of the Coupling Condition clearly holds for elements of the strong operator closure of $B$, which by von Neumann's double commutant theorem is just $B^{\prime \prime}$. Thus it would hold for $b=e$. But then we would have

$$
\begin{aligned}
& \langle x, d x\rangle=\langle x, e w\rangle=-\left\langle e^{*} w, x\right\rangle \\
& =-\langle w, e x\rangle=-\langle w, w-d w\rangle=\langle w,(d-1) w\rangle \leqq 0 .
\end{aligned}
$$


From this it would follow that $d x=0$ and $(d-1) w=0$. From the latter it would follow that $d$ acts as the identity on $A w$. But then from the former it would follow that $x$ is orthogonal to $A w$, contrary to assumption.

Q.E.D.

We remark that some statement concerning the identity operator being in the strong operator closures of the algebras is necessary for the proof of the converse statement, as can be seen by considering the case in which $A$ consists of all operators on $V$ and $B$ consists of the zero operator alone.

That the question of when two algebras of operators generate each others commutants can be a fairly delicate question may be seen by considering the "factorizations which are not coupled factors" found by Murray and von Neumann (Sections 3.1 and 13.4 of [13]). This question is closely related to the subject of normalcy in von Neumann algebras (see references in [21]).

\section{Duality}

Let $G$ be a real Hilbert space, let $H$ be its complexification, and let $V$ be the Fock space over $G$ (p. 18 of [11]), that is,

$$
V=\oplus H^{(p)}
$$

where $H^{(p)}$ denotes the Hilbert space of symmetric tensors of order $p$ over $H$. In particular, $H^{(0)}$ is taken to be the complex numbers, and we will let $w$ denote the number 1 from this space, viewed as an element of $V$ (the Fock vacuum). Then the Weyl form of the Fock representation of $H$ on $V$ (p. 172 of [11]) is the projective unitary representation, $F$, of $H$ on $V$ having the properties that for $h, k \in H$

$$
F(h+k)=F(h) F(k) \exp (-i \operatorname{Im}\langle h, k\rangle),
$$

where Im denotes "imaginary part of", and

$$
F(h) w=\exp \left(-\|h\|^{2} / 2\right) \Sigma(p !)^{-\frac{1}{2}} h^{(p)},
$$

where in the sum $p$ ranges over the non-negative integers, and $h^{(p)}$ is the tensor $h \times \cdots \times h$ of order $p$. (We will use the symbol $\times$ to denote symmetric tensor products.) To pass to the frequently used definition in which there are additional factors of 2, (as in [10]), it suffices to pass from $h$ to $\sqrt{2} h$ in the above.

We will use the following facts about Fock space several times. First, any elementary symmetric tensor, $h_{1} \times \cdots \times h_{p}$, can be expressed as a finite sum of tensors of the form $h^{(p)}$ where the $h$ 's involved are real linear combinations of the $h_{j}$ (see the footnote on p. 199 of [3] or the 
equation for polynomials near the top of p. 176 of [14]). Secondly, if, for fixed $h \in H$, we take the $p$-th derivative at 0 of the function

$$
t \rightarrow \exp \left(\|t h\|^{2} / 2\right) F(t h) w
$$

as $t$ ranges over the real numbers, it is easily seen that we obtain $(p !)^{\frac{1}{2}} h^{(p)}$ (p. 21 of [11]). From this it follows almost immediately that, for fixed $h \in H$, the closed subspace of $V$ spanned by the vectors $F(t h) w$ as $t$ ranges over the real numbers, meets $H^{(p)}$ exactly in the one-dimensional subspace spanned by $h^{(p)}$.

Now in $[1,2,15]$ it is shown that duality for a free Bose field comes down to the following situation. Let $J$ and $K$ be arbitrary (closed) subspaces of $G$, and let $J^{\perp}$ and $K^{\perp}$ denote their orthogonal complements in $G$. Let $M$ and $N$ denote the real subspaces $J+i K$ and $K^{\perp}+i J^{\perp}$ of $H$. Note that if $m \in M, n \in N$, then $\langle m, n\rangle$ is real, so that $F(m) F(n)=F(n) F(m)$ Let $A=R(J, K)=R(M)$ be the von Neumann algebra of operators on $V$ generated by $\{F(m): m \in M\}$, and let $B=R\left(K^{\perp}, J^{\perp}\right)=R(N)$ be the von Neumann algebra generated by $\{F(n): n \in N\}$. Then the relation in the previous sentence shows that $a b=b a$ for $a \in A, b \in B$. Duality then becomes the assertion that $A^{\prime}=B$ (and so $B^{\prime}=A$ ).

We would like to use the commutation theorem of the first section to prove this assertion of duality. Since initially we know little about $A^{\prime}$ and $B^{\prime}$, we will, in accordance with the remark made immediately after the statement of the commutation theorem, first determine when $A w+B w$ is dense in $V$. It turns out that in the process we obtain a very simple proof of part of the special case of the Reeh-Schlieder theorem [16] which applies to a free Bose field (Lemma 7 of [2], Lemma 1 of [9], or p. 290 of $[10])$.

Proposition. If $J+K$ is dense in $G$ then $A w$ is dense in $V$ (part of the Reeh-Schlieder theorem). Conversely, if $A w+B w$ is dense in $V$, then either $J+K$ or $J^{\perp}+K^{\perp}$ is dense in $G$.

Proof. Suppose that $J+K$ is dense in $G$. Then $M+i M$ is clearly dense in $H$. Let $W$ be the closure of $A w$ in $V$. Then $W$ will contain $F(m) w$ for any $m \in M$, and so, as discussed above, will contain $m^{(p)}$. It follows, again as discussed above, that $W$ contains any tensor of the form $m_{1} \times---\times m_{p}$ for $m_{j} \in M$. Since $W$ is a complex subspace, it follows that it contains any tensor of the form

$$
\left(m_{1}+i m_{1}^{\prime}\right) \times \cdots \times\left(m_{p}+i m_{p}^{\prime}\right)
$$

for $m_{j}, m_{j}^{\prime} \in M$. But $M+i M$ is dense in $H$, and from this it follows that $W$ contains $H^{(p)}$ for each $p$, and so $W=V$.

Conversely, suppose that neither $J+K$ nor $J^{\perp}+K^{\perp}$ is dense in $G$. Let $f$ and $g$ be elements of $G$ which are orthogonal to $J+K$ and $J^{\perp}+K^{\perp}$ 
respectively. Then it is easily seen that $f \times g$ will be orthogonal to $A w+B w$, so that $A w+B w$ is not dense in $V$. Q.E.D.

As is shown in $[2,15]$, the subspaces $J$ and $K$ which actually arise in considering a free Bose field are always in general position, that is, $J, K, J^{\perp}, K^{\perp}$ are pairwise disjoint. In particular $J+K$ and $J^{\perp}+K^{\perp}$ will both be dense in $G$, and so the above Proposition is applicable. This shows that it is reasonable to try to apply the Commutation Theorem of the first section, since the situation will be decided by determining whether the Coupling Condition is true for all $x \in V$. In more complicated situations in which one might want to prove duality, if the ReehSchlieder theorem holds, then this again means that it is reasonable to try to apply the Commutation Theorem.

We now proceed to prove that duality holds for free Bose fields.

Main Theorem. Let the notation be as above. If $J$ and $K$ are real subspaces of $G$ such that $J+K$ is dense in $G$, then the Coupling Condition of the Commutation Theorem holds, and so $A^{\prime}=B$.

Proof. Let $x \in V$, and suppose it satisfies Eqs. (1) and (2) of the Coupling Condition. Since $A$ and $B$ are generated by the $F(m)$ and $F(n)$ respectively, and since $F(m)^{*}=F(-m)$, it follows that we have

$$
\begin{aligned}
& \langle x, F(m) w\rangle=+\langle F(-m) w, x\rangle \text { for } m \in M, \\
& \langle x, F(n) w\rangle=-\langle F(-n) w, x\rangle \text { for } n \in N \text {. }
\end{aligned}
$$

Replacing $m$ and $n$ by $t m$ and $t n$ for real $t$, and taking the $p$-th derivative at 0 , we obtain, as discussed above,

$$
\begin{array}{lll}
\left\langle x, m^{(p)}\right\rangle=+\left\langle(-1)^{p} m^{(p)}, x\right\rangle & \text { for } & m \in M \\
\left\langle x, n^{(p)}\right\rangle=-\left\langle(-1)^{p} n^{(p)}, x\right\rangle & \text { for } & n \in N
\end{array}
$$

for each non-negative integer $p$. Let $M^{(p)}$ and $N^{(p)}$ denote the real subspaces of $H^{(p)}$ spanned by the $m^{(p)}$ and the $n^{(p)}$ respectively. Then it follows from the above that we have

$$
\begin{array}{ll}
\langle x, u\rangle=+\left\langle u,(-1)^{p} x\right\rangle & \text { for } \quad u \in M^{(p)}, \\
\langle x, v\rangle=-\left\langle v,(-1)^{p} x\right\rangle & \text { for } \quad v \in N^{(p)} .
\end{array}
$$

If we multiply the second equation by $i$ and subtract, we obtain

$$
\langle x, u+i v\rangle=\left\langle u+i v,(-1)^{p} x\right\rangle
$$

for all $u \in M^{(p)}$ and $v \in N^{(p)}$. What we will show is that the $u+i v$ are dense in $H^{(p)}$, so that it will follow that

$$
\langle x, v\rangle=\left\langle v,(-1)^{p} x\right\rangle
$$


for all $v \in H^{(p)}$. But since $H^{(p)}$ is a complex space, this can only be true if the component of $x$ in $H^{(p)}$ is 0 . Since this will be true for all $p$, it will follow that $x=0$, as desired. Thus the proof of duality comes down to proving:

Lemma. Let $p$ be fixed. Then the real subspace $M^{(p)}+i N^{(p)}$ is equal to $H^{(p)}$.

The proof of this lemma seems to be slightly delicate. Our tactics will be as follows: Define a real inner product, $\langle,\rangle_{r}$ on $H$ by

$$
\langle f, g\rangle_{r}=\operatorname{Re}(\langle f, g\rangle),
$$

and similarly for $H^{(p)}$, so they become real Hilbert spaces (but note that as such $H^{(p)}$ is not the real $p$-th symmetric power of the real space $H$ ). View multiplication by $i$ as an orthogonal operator on each. Now for $m \in M, n \in N$ we know that $\langle m, n\rangle$ is real (it is because of this that $A$ and $B$ commute). It follows that $\left\langle m^{(p)}, n^{(p)}\right\rangle$ is real, and so

$$
\left\langle m^{(p)}, i n^{(p)}\right\rangle_{r}=0 \text {. }
$$

Thus $M^{(p)}$ and $i N^{(p)}$ are real orthogonal. Since $M^{(p)}$ and $i N^{(p)}$ are closed, it follows that to show that $M^{(p)}+i N^{(p)}=H^{(p)}$, it suffices to show that it is dense in $H^{(p)}$. We wish to place ourselves in such a position that we can use the following fact (in which the symbol $\oplus$ means orthogonal direct sum).

Sublemma 1. Let $X$ be a real Hilbert space, let $j$ be an orthogonal operator on $X$, and let $Y$ and $Z$ be two subspaces of $X$. Suppose that

(1) $Y$ and $Z$ are orthogonal,

(2) $Y+j Y$ is a closed subspace, $U$, of $X$,

(3) $(Z+j Z) \oplus(Y \cap j Y)=U$. Then $Y \oplus Z=U$.

Proof. Since $Y$ is orthogonal to $Z$, it follows that $Y \oplus Z$ is closed (and contained in $U$ by (3)). Note that $Z+j Z$ is in fact orthogonal to $Y \cap j Y$ (so that the hypotheses also imply that $Z+j Z$ will be closed). Let $u$ be an element of $U$ which is orthogonal to $Y+Z$. According to (3) $u=z+j z^{\prime}+v$ where $z, z^{\prime} \in Z, v \in Y \cap j Y$. Since $u$ is orthogonal to $Y$, and so to $v$, as is $z+j z^{\prime}$, it follows that $v=0$. According to (2) we have $u=y+j y^{\prime}$ for $y, y^{\prime} \in Y$. Then

$$
\begin{aligned}
& \langle u, u\rangle=\left\langle u, z+j z^{\prime}\right\rangle=\left\langle u, j z^{\prime}\right\rangle \\
& =\left\langle y+j y^{\prime}, j z^{\prime}\right\rangle=\left\langle y, j z^{\prime}\right\rangle=\langle y, u-z\rangle=0 .
\end{aligned}
$$

Thus $u=0$, and so $Y+Z=U$. Q.E.D.

Unfortunately this sublemma is no longer true if we do not assume that $Y+j Y$ and $Z+j Z$ are closed. This can be seen, as was pointed out 
to me by Robert T. Powers, by embroidering on the situation obtained by letting $X=L^{2}(R), j$ be Fourier transform, $Y=L^{2}([1, \infty])$ and $Z=\left(L^{2}([-\infty, 0])\right)$. It is because of this that we need to cut down $M$ and $N$ so that we can work with subspaces whose sum is closed. This is accomplished by:

Sublemma 2. Let $J$ and $K$ be subspaces of a real Hilbert space $G$ such that $J+K$ is dense in $G$. Then there is a sequence, $\left\{e_{r}\right\}$, of projections on $G$, converging in the strong operator topology to the identity operator on $G$, such that for every $r$

(1) $e_{r} J \cong J$ and $e_{r} K \cong K$,

(2) $e_{r} J+e_{r} K=e_{r} G$ (and so is closed),

(3) $e_{r} J^{\perp}+e_{r} K^{\perp}$ is closed.

Proof. Let $P=\left(J \cap K \oplus J^{\perp} \cap K \oplus J \cap K^{\perp}\right)^{\perp}$. Then

$$
\begin{aligned}
& J=J \cap K \oplus J \cap K^{\perp} \oplus J_{1} \\
& K=J \cap K \oplus J^{\perp} \cap K \oplus K_{1}
\end{aligned}
$$

where $J_{1}, K_{1}$ are subspaces of $P$ which are now in general position. Then according to a theorem of Halmos (Theorem 3 of [12]) $P$ can be written as $Q \oplus Q$, and there is a positive contraction, $s$, from $Q$ to itself with kernel 0 , such that

$$
J_{1}=\{(q, s(q)): q \in Q\}, \quad K_{1}=\{(q,-s(q)): q \in Q\} .
$$

Let $\left\{d_{r}\right\}$ be the resolution of the identity for $s$, and let $e_{r}=1-d_{(1 / r)}$, but now only for $r$ a positive integer. Extend $e_{r}$ to $P$ by letting it act on each copy of $Q$, and then extend it to $G$ by letting it be the identity on $P^{\perp}$. Then $s$ will be invertible on $e_{r} Q$ for each integer $r$, and from this it is easily seen that $e_{r} J_{1}+e_{r} K_{1}=e_{r} P$. From this it follows that $e_{r} J+e_{r} K$ $=e_{r} G$. Similar simple arguments verify the remaining assertions. Q.E.D.

For notational simplicity we now fix an $r$, and let $J$ and $K$ denote $e_{r} J$ and $e_{r} K$. What we now know about $J$ and $K$ is that $J+K$ is a closed subspace, say $L$, of $G$, and that $\left(J^{\perp} \cap L\right)+\left(K^{\perp} \cap L\right)$ is a closed subspace of $L$ [because $\left(e_{r} J\right)^{\perp} \cap L=e_{r}\left(J^{\perp}\right)$ in the previous notation]. Furthermore it is clear that

$$
\left(J^{\perp} \cap L\right)+\left(K^{\perp} \cap L\right) \oplus J \cap K=L .
$$

Now let $M=J+i K, N=\left(K^{\perp} \cap L\right)+i\left(J^{\perp} \cap L\right)$ (which are just $e_{r}$ applied to the previous $M$ and $N$ ). Then from the above facts it follows that $M+i M=\mathbb{C} L,(N+i N)$ is closed in $H$, and $N+i N \oplus(M \cap i M)=\mathbb{C} L$, where $\mathbb{C} L$ denotes the closed subspace $L+i L$ in $H$. Also, as before, $\langle m, n\rangle$ is real for $m \in M, n \in N$.

We now need to lift these facts to $H^{(p)}$. For this we need: 
Sublemma 3. Let $H$ be a complex Hilbert space, and let $M$ be a real subspace of $H$ such that $M+i M$ is closed in $H$. Then $M^{(p)}+i M^{(p)}$ $=(M+i M)^{(p)}$. In particular, $M^{(p)}+i M^{(p)}$ is closed in $H^{(p)}$.

Proof. Let $D=M+i M$. It is a triviality that $M^{(p)}+i M^{(p)}$ is dense in $D^{(p)}$, so the crux of the matter is to show that it is closed.

Since $M \cap i M$ is complex, it will have the same orthogonal compliment in $H$ whether $H$ is viewed as complex or real. Let $M^{\prime}=(M \cap i M)^{\perp} \cap M$, so that $M^{\prime} \subseteq M, i M^{\prime} \cap M=\{0\}$, and $M+i M^{\prime}=D$. On $M+i M^{\prime}$ define a new norm by $\left\|m+i m^{\prime}\right\|=\|m\|+\left\|m^{\prime}\right\|$. Then $M+i M^{\prime}$ is clearly a Banach space for this norm, and the obvious bijection of $M+i M^{\prime}$ onto $D$ is continuous. Since $D$ is closed and so is a Banach space, the open mapping theorem is applicable to this bijection, so that its inverse is continuous. This means that the real linear projection, $P$, of $D$ onto $M$ along $i M^{\prime}$ is continuous, as is $Q=I_{D}-P$, the projection of $D$ onto $i M^{\prime}$ along $M$ (where $I_{D}$ denotes the identity operator on $D$ ). We extend $P$ and $Q$ to $H$ by letting them be zero on $D^{\perp}$.

Consider $H$ as a real Hilbert space, and denote the corresponding real $p$-th symmetric power by $H^{[p]}$. Since we have $I_{D}=P+Q$, we have $I_{D}^{[p]}=\Sigma E_{k}$, where

$$
E_{k}=\left(\begin{array}{l}
p \\
k
\end{array}\right) P^{[k]} \times Q^{[p-k]}, \quad 1 \leqq k \leqq p .
$$

Now it is easily seen that if $k \neq j$ then $E_{j} E_{k}=0$. Since $I_{D}^{[p]}$ is the identity operator on $D^{[p]}$ it follows that each $E_{k}$ is a projection (not necessarily self-adjoint). It follows that $D^{[p]}$ is the sum of the ranges of these projections.

Now the real linear mapping, $T$, from $H^{[p]}$ to $H^{(p)}$ which carries $h^{[p]}$ to $h^{(p)}$ is continuous (but not norm-decreasing) and surjective. This assertion is easily verified by choosing an orthonormal basis $\left\{b_{j}\right\}$ for $H$ as a complex space, and using the fact that $\left\{b_{j}, i b_{j}\right\}$ is then an orthonormal basis for $H$ as a real space. Since $D$ is a closed complex subspace of $H$, the same arguments show that $T$ carries $D^{[p]}$ onto $D^{(p)}$. Thus $G^{(p)}$ will be the sum of the images under $T$ of the ranges of the $E_{k}$.

Now the elementary tensors of the form

$$
m_{1} \times---\times m_{k} \times i m_{1}^{\prime} \times \cdots \times i m_{p-k}^{\prime}
$$

where the $m_{j} \in M, m_{j}^{\prime} \in M^{\prime} \cong M$, clearly span a dense subspace of the range of $E_{k}$. But $T$ carries such an elementary tensor into either $M^{(p)}$ or $i M^{(p)}$, depending upon whether $p-k$ is even or odd. It follows that $T$ carries the range of $E_{k}$ into $M^{(p)}$ or $i M^{(p)}$, and consequently $M^{(p)}+i M^{(p)}$ $=G^{(p)}$ as desired. Q.E.D.

We now return to the proof of the Lemma, but maintaining the notation introduced right after Sublemma 2. It follows from Sublemma 3 
that $M^{(p)}+i M^{(p)}=(\mathbb{C} L)^{(p)}$, and that

But

$$
N^{(p)}+i N^{(p)}=\left((M \cap i M)^{\perp} \cap \mathbb{C} L\right)^{(p)} .
$$

$$
\left((M \cap i M)^{\perp} \cap \mathbb{C} L\right)^{(p)}+M^{(p)} \cap i M^{(p)}
$$

is dense in $(\mathbb{C} L)^{(p)}$, because any element of $\mathbb{C} L$ is of the form $m+m^{\prime}$ where $m \in M \cap i M$ and $m^{\prime} \in(M \cap i M)^{\perp} \cap \mathbb{C} L$, so $\left(m+m^{\prime}\right)^{(p)}$ is a real linear combination of tensors of the form $m^{(k)} \times m^{\prime(p-k)}$. But if $k=0$ then such a tensor is in $\left((M \cap i M)^{\perp} \cap \mathbb{C} L\right)^{(p)}$, whereas if $k \geqq 1$ then $m^{\prime}$ can be written as $m^{\prime}+i m^{\prime \prime}$ for $m^{\prime}, m^{\prime \prime} \in M$, and in the above tensor the $i$ 's can be taken in and out of the $m$ factors, so that the tensor is clearly in $M^{(p)} \cap i M^{(p)}$. It follows that

$$
N^{(p)}+i N^{(p)} \oplus M^{(p)} \cap i M^{(p)}=(\mathbb{C} L)^{(p)} .
$$

Setting $y=M^{(p)}$ and $Z=i N^{(p)}$, we see that the conditions of Sublemma 1 are fulfilled, and so we can conclude that

$$
M^{(p)}+i K^{(p)}=(\mathbb{C} L)^{(p)} .
$$

Recalling that we had changed notation after Sublemma 2, we can write this in terms of the original $M$ and $N$ as

$$
\left(e_{r} M\right)^{(p)}+i\left(e_{r} N\right)^{(p)}=\left(e_{r} H\right)^{(p)} .
$$

Since $e_{r}$ converges strongly to the identity operator on $H$, it follows that $M^{(p)}+i K^{(p)}$ is dense in $H^{(p)}$ as desired. Q.E.D.

Setting $J=K=G$ in the above Theorem, we immediately obtain:

Corollary. The Fock representation is irreducible.

In the case in which neither $J+K$ nor $J^{\perp}+K^{\perp}$ is dense in $G$, the Main Theorem of this section remains true, but our Commutation Theorem does not seem directly applicable. Instead one must first make a simple reduction, which is discussed in Section 5 of [1].

Acknowledgements. I would like to thank Richard H. Herman, Richard V. Kadison, Robert T. Powers, Shoichiro Sakai, E. James Woods, and Alfons Van Daele for helpful conversations concerning this subject. The research for this paper was conducted while I was visiting at the University of Pennsylvania, and I would like to express my appreciation to the members of the Department of Mathematics there for their warm hospitality during my visit. This research was partly supported by National Sciences Foundation grant GP-30798X.

\section{References}

1. Araki,H.: A lattice of von Neumann algebras associated with the quantum theory of a free Bose field. J. Math. Phys. 4, 1343-1362 (1963)

2. Araki,H.: Von Neumann algebras of local observables for free scalar field. J. Math. Phys. 5, 1-13 (1964) 
3. Araki,H., Woods, E.J.: Complete Boolean algebras of type I factors. Publ. R.I.M.S. Kyoto U. 157-242 (1966)

4. Dell'Antonio, G.F.: Structure of the algebras of some free systems, Commun. math. Phys. 9, 81-117 (1968)

5. Dixmier, J.: Les Algèbres d'Opérateurs dans l'Espace Hilbertien, $2 d$ ed., Paris: Gauthier-Villars 1969

6. Doplicher, S., Haag, R., Roberts, J.E.: Fields, observables and gauge transformations, II, Commun. math. Phys. 15, 173-200 (1969)

7. Doplicher,S., Roberts, J.E.: Fields, statistics and non-Abelian gauge groups. Commun. math. Phys. 28, 331-348 (1972)

8. Dunford, N., Schwartz,J.: Linear operators, vol. II. New York: Interscience 1963

9. Eckmann,J.-P., Osterwalder,K.: An application of Tomita's theory of modular Hilbert algebras: Duality for free Bose fields. J. Funct. Anal. 13, 1-12 (1973)

10. Emch, G.G.: Algebraic methods in statistical mechanics and quantum field theory. New York: Wiley-Interscience 1972

11. Guichardet,A.: Symmetric Hilbert spaces and related topics. Lecture Notes in Mathematics 261, Berlin-Heidelberg-New York: Springer 1972

12. Halmos, P.R.: Two subspaces. Trans. Am. Math. Soc. 144, 381-389 (1969)

13. Murray, F. J., von Neumann, J.: On rings of operators, Ann. Math. 37, 116-229 (1936)

14. Nelson,E.: A functional calculus for non-commuting operators. Functional Analysis and Related Fields, ed. by Felix E. Browder, Berlin-Heidelberg-New York: Springer 1970

15. Osterwalder, K.: Duality for free Bose fields. Commun. math. Phys. 29, 1-14 (1973)

16. Reeh,H., Schlieder,S.: Bemerkungen zur Unitarequivalenz von Lorentzvarianten Feldern. Nuovo Cimento 26, 32- 42 (1961)

17. Rieffel,M., Van Daele,A.: The commutation theorem for tensor products of von Neumann algebras. Bull. London Math. Soc. To appear

18. Sakai, S.: On the tensor product of $W^{*}$-algebras. Am. J. Math. 90, 335-341 (1968)

19. Sakai, S.: $C^{*}$-algebras and $W^{*}$-algebras. Berlin-Heidelberg New York: Springer 1971

20. Takesaki,M.: Tomita's theory of Modular Hilbert algebras and its applications. Springer Lecture Notes no. 128, Berlin-Heidelberg-New York: Springer 1970

21. Vowden, B.J.: Normalcy in von Neumann algebras. Proc. London Math. Soc. 27, $88-100(1973)$

Communicated by H. Araki

Marc A. Rieffel

Department of Mathematics

University of California

Berkeley, Calif. 94720, USA 